\title{
FROM FREUDENTHAL'S SPECTRAL THEOREM TO PROJECTABLE HULLS OF UNITAL ARCHIMEDEAN LATTICE-GROUPS, THROUGH COMPACTIFICATIONS OF MINIMAL SPECTRA
}

\author{
RICHARD N. BALL, VINCENZO MARRA, DANIEL MCNEILL, AND ANDREA PEDRINI
}

\begin{abstract}
We use a landmark result in the theory of Riesz spaces - Freudenthal's 1936 Spectral Theorem - to canonically represent any Archimedean lattice-ordered group $G$ with a strong unit as a (non-separating) lattice-group of real valued continuous functions on an appropriate $G$-indexed zero-dimensional compactification $w_{G} Z_{G}$ of its space $Z_{G}$ of minimal prime ideals. The two further ingredients needed to establish this representation are the Yosida representation of $G$ on its space $X_{G}$ of maximal ideals, and the well-known continuous surjection of $Z_{G}$ onto $X_{G}$. We then establish our main result by showing that the inclusion-minimal extension of this representation of $G$ that separates the points of $Z_{G}$ - namely, the sublattice subgroup of $\mathrm{C}\left(Z_{G}\right)$ generated by the image of $G$ along with all characteristic functions of clopen (closed and open) subsets of $Z_{G}$ which are determined by elements of $G$ - is precisely the classical projectable hull of $G$. Our main result thus reveals a fundamental relationship between projectable hulls and minimal spectra, and provides the most direct and explicit construction of projectable hulls to date. Our techniques do require the presence of a strong unit.
\end{abstract}

\section{INTRODUCTION}

In 1936, Freudenthal proved his well-known Spectral Theorem 11 for Riesz spaces (real linear spaces with a compatible lattice order) with motivations coming from the theory of integration. (See [17, 40.2] for a handbook treatment.)

In its basic version, the theorem asserts that any element of a Riesz space $R$ with a strong unit $u$ and the principal projection property may be uniformly approximated, in the norm that $u$ induces on $R$, by abstract characteristic functions - "components of the unit $u$ ". See Subsection 2.1 for more details. Freudenthal's theorem led to a considerable amount of research on Riesz spaces and their generalisations, the lattice-ordered Abelian groups that concern us here, and which we call $\ell$-groups for short. (For background we refer to [17, 10, 13].) One main line of research concentrated on extending one given structure $G$ to a minimal completion that enjoys the principal projection property, where Freudenthal's theorem therefore applies. Such an extension is called the projectable hull of $G$; please see Subsection 2.2 for details.

In 1973, Conrad 7] proved the existence and uniqueness of projectable hulls of (a class of lattice-groups more general than) Archimedean $\ell$-groups, using his previous

Key words and phrases. Freudenthal's Spectral Theorem, Riesz space, Lattice-ordered group, Archimedean property, Yosida representation, Principal projection property, Projectable hull, Spectral space, Maximal ideal, Minimal ideal, Zero-dimensional space, Compactification.

2010 Mathematics Subject Classification. Primary: 06F20. Secondary: 54D35. 
construction in [6] of the essential closure of such an $\ell$-group - the largest extension of the structure that is essential, in the sense recalled in Subsection 2.2. At about the same time, Chambless [5] exhibited a different construction of the projectable hull based on direct limits; cf. also Bleier's construction in [4. Here we present a new construction of the projectable hull of an Archimedean $\ell$-group equipped with a strong order unit $u$ - an element whose multiples eventually dominate any other element in the $\ell$-group - that does not use direct limits, nor essential closures. Our construction exposes instead the intimate connection between projectable hulls and zero-dimensional compactifications of spectral spaces of minimal prime ideals. Closing the circle of ideas beginning with Freudenthal, to establish this connection we will need to apply his Spectral Theorem at a key step of the construction. We now recall some standard notions, and introduce notations that will remain in force throughout the paper.

Throughout, all lattice-ordered groups are Abelian, and referred to simply as $\ell$-groups for short. We write $\mathbf{U}$ for the category whose typical object is a pair $(G, u)$, where $G$ is an $\ell$-group that is Archimedean - whenever $0 \leqslant n g \leqslant h$ for $h, g \in G$ and all integers $n \geqslant 1$, then $g=0$ - equipped with a distinguished (strong order) unit $u \in G$ - an element $u \geqslant 0$ such that for all $g \in G$ there is an integer $n \geqslant 1$ such that $n u \geqslant g$. As morphisms, we take the lattice-group homomorphisms ( $\ell$-homomorphisms) that are unital, i.e. preserve the distinguished units. It will transpire that our techniques do require the existence of a strong unit, as opposed to the existence of a weak unit. Recall that a weak (order) unit of $G$ is an element $w \in G^{+}$such that for each $g \in G, w \wedge|g|=0$ implies $g=0$. Here, $|g|:=(g \vee 0)+(-g \vee 0)$ is the absolute value of $g$.

By an ideal in an $\ell$-group we mean, as usual, a sublattice subgroup $I$ of $G$ that is order-convex: whenever $a, c \in I, b \in G$, and $a \leqslant b \leqslant c$, then $b \in G$. Ideals are exactly the kernels of (unital) $\ell$-homomorphisms, i.e. the morphisms in the unrestricted category of abelian $\ell$-groups, and the usual homomorphism theorems hold. An ideal $\mathfrak{p}$ of $G$ is prime if, and only if, it is proper $(\mathfrak{p} \neq G)$ and the quotient $\ell$-group $G / \mathfrak{p}$ is totally ordered. A prime ideal is maximal if it is inclusion-maximal - equivalently, if $G / \mathfrak{p}$ is non-trivial and simple, i.e. it has no non-trivial proper ideals. Ideals that are inclusion-maximal are automatically prime. A prime ideal is minimal if it is inclusion-minimal. For any unital $\ell$-group $(G, u)$, we denote by Max $G$ the collection of its maximal (prime) ideals, and by Min $G$ the collection of its minimal prime ideals. We topologize both $\operatorname{Max} G$ and $\operatorname{Min} G$ using the spectral, or Zariski topology. The closed sets for this topology are given by subsets of the form

$$
\mathbb{V}_{M}(A):=\{\mathfrak{m} \in \operatorname{Max} G \mid \mathfrak{m} \supseteq A\}
$$

and

$$
\mathbb{V}_{m}(A):=\{\mathfrak{p} \in \operatorname{Min} G \mid \mathfrak{p} \supseteq A\},
$$

as $A$ ranges over arbitrary subsets of $G$. The resulting topological spaces are called the maximal and minimal prime spectrum of $G$, respectively. The topology on $\operatorname{Max} G$ is also called the hull-kernel topology, because it agrees with the classical hull-kernel topology for rings of continuous functions [12, mutatis mutandis. Accordingly, we call $\mathbb{V}_{M}(A)$ (or $\left.\mathbb{V}_{m}(A)\right)$ the zero set of $A$ (on the appropriate space), and its complement - denoted by $\mathbb{S}_{M}(A)\left(\right.$ or $\left.\mathbb{S}_{m}(A)\right)$ - the support of $A$. It is 
known [3, 10.2.1] that the collections

$$
\left\{\mathbb{V}_{M}(\{g\})\right\}_{g \in G} \quad \text { and } \quad\left\{\mathbb{V}_{m}(\{g\})\right\}_{g \in G}
$$

form a closed base for $\operatorname{Max} G$ and $\operatorname{Min} G$, respectively. Throughout we write $\mathbb{V}_{M}(g)$ in place of $\mathbb{V}_{M}(\{g\})$, and similarly for $\mathbb{V}_{m}, \mathbb{S}_{M}$, and $\mathbb{S}_{m}$.

The space Max $G$ is a Hausdorff space that is compact precisely because of the assumption that $G$ has a (strong) unit $u$; see [3, 10.2.5]. The space Min $G$ is a Hausdorff zero-dimensional space that need not be compact [3, 10.2.1]. Whether it is or not has nothing to do with the existence of a strong unit, but rather with complementation properties of the lattice $G^{+}:=\{g \in G \mid g \geqslant 0\}$, the positive cone of $G$; see Section 3 .

Notation. For the rest of this paper, we let $(G, u)$ denote a $\mathbf{U}$-object, and set

$$
\begin{aligned}
X_{G} & :=\operatorname{Max} G, \\
Z_{G} & :=\operatorname{Min} G .
\end{aligned}
$$

If $X$ is any topological space, always at least Tychonoff, we write $\mathrm{C}(X)$ for the $\ell$-group of continuous functions $X \rightarrow \mathbb{R}$ under pointwise operations. If $X$ is compact, the function $1_{X}$ constantly equal to 1 over $X$ is a strong unit of $\mathrm{C}(X)$ by the Extreme Value Theorem. We always tacitly consider $\mathrm{C}(X)$ endowed with the distinguished unit $1_{X}$, and hence as a U-object when $X$ is compact Hausdorff. The classical Yosida representation [21] of $(G, u)$ yields a canonical unital lattice-group embedding $\widehat{\imath}: G \hookrightarrow \mathrm{C}\left(X_{G}\right)$; details are recalled in Subsection 2.3 .

It is well known that $Z_{G}$ is canonically thrown onto $X_{G}$, as follows. Given $\mathfrak{a} \in Z_{G}$, a standard argument [17, 27.4] shows that, by virtue of the presence of the (strong) unit $u$, there exists at least one $\mathfrak{m}_{\mathfrak{a}} \in X_{G}$ such that $\mathfrak{a} \subseteq \mathfrak{m}_{\mathfrak{a}}$. Since the prime ideals of $G$ form a root system under set-theoretic inclusion [3, 2.4.3] — that is, the set of prime ideals containing any given prime ideal is linearly ordered such an $\mathfrak{m}_{\mathfrak{a}}$ must be unique; in other words, the set $\uparrow \mathfrak{a} \cap X_{G}$ is a singleton, where $\uparrow \mathfrak{a}:=\{\mathfrak{b} \subseteq G \mid \mathfrak{a} \subseteq \mathfrak{b}, \mathfrak{b}$ a prime ideal $\}$. Hence there is a function

$$
\lambda: Z_{G} \rightarrow X_{G}
$$

defined by

$$
\mathfrak{a} \in Z_{G} \stackrel{\lambda}{\longmapsto} \mathfrak{m}_{\mathfrak{a}} \in X_{G}
$$

By [3, 10.2.5], the map $\lambda$ is continuous, and is a surjection by the standard fact that each prime ideal contains a minimal prime ideal [3, 2.4.5].

Remark 1.1. The strong unit is crucial here. There exist non-trivial Archimedean (and even Dedekind-complete) $\ell$-groups with a weak unit and no maximal ideal at all; see [17, 27.8]. Thus, while the Yosida representation has an important extension to $\ell$-groups with a weak unit [22, 15], the existence of the map (1), a key ingredient to our construction, does require a strong unit. A generalisation of our results to the case of weak units would surely be of interest, but it would require substantial modifications.

Composition of the map $\lambda$ with the Yosida representation of $G$ embeds $G$ as a unital sublattice-subgroup ( $\ell$-subgroup) into $\mathrm{C}\left(Z_{G}\right)$ : one sends $g \in G$ to $\widehat{g} \circ$ $\lambda: Z_{G} \rightarrow \mathbb{R}$. The assignment is injective because $\lambda$ is surjective. In Section 3 this observation is considerably strengthened. It turns out that $G$ determines a specific zero-dimensional compactification of its minimal spectrum which we denote 
$w_{G} Z_{G}$. The clopen subsets of $w_{G} Z_{G}$, we will see, are (finitely) generated from the clopen subsets of $Z_{G}$ of the form $\mathbb{V}_{m}(g)$. (Please see Section 3 for details on this compactification.) We prove in Theorem 3.11 that $G$ embeds as a unital $\ell$-subgroup of $\mathrm{C}\left(w_{G} Z_{G}\right)$. We will see that this stronger embedding of $G$ is in fact granted by Freudenthal's Spectral Theorem. Now, by the Yosida theory (see again Subsection 2.3), the image of $G$ in $\mathrm{C}\left(w_{G} Z_{G}\right)$ does not separate the points of the base space, unless $X_{G}$ and $w_{G} Z_{G}$ are homeomorphic. We can however consider a minimal extension of the image of $G$ inside $\mathrm{C}\left(w_{G} Z_{G}\right)$ which separates the points. Indeed, since $w_{G} Z_{G}$ is zero-dimensional, there is a canonical such extension: we must adjoin to the image of $G$ all characteristic functions of clopen subsets of $w_{G} Z_{G}$. We thereby obtain a unital embedding

$$
\pi_{G}: G \hookrightarrow \mathscr{P}(G),
$$

where $\mathscr{P}(G)$ denotes the unital $\ell$-subgroup of $\mathrm{C}\left(w_{G} Z_{G}\right)$ generated by the representation of $G$ into $\mathrm{C}\left(w_{G} Z_{G}\right)$, together with all characteristic functions $w_{G} Z_{G} \rightarrow \mathbb{R}$ - the continuous maps with range contained in $\{0,1\}$. We now have the homeomorphism $\operatorname{Max} \mathscr{P}(G) \cong w_{G} Z_{G}$. In Theorem 4.5 we show that the elements of $\mathscr{P}(G)$ may be characterised amongst elements of $\mathrm{C}\left(w_{G} Z_{G}\right)$ as those functions with the property that, for an appropriate finite partition of $w_{G} Z_{G}$ into clopens, they agree with the image of some element of $G$ locally at each clopen. Building on this we finally show in Theorem [5.1 that (3) is the projectable hull of $(G, u)$, thus obtaining our main result. Summarising, we prove the existence of the projectable hull of any $\mathbf{U}$-object $(G, u)$ by exhibiting it as a natural substructure of $\mathrm{C}\left(w_{G} Z_{G}\right)$, namely, $\mathscr{P}(G)$.

Several intermediate results in this paper admit a fuller development of considerable potential interest. We focus here on the proof of our main Theorem 5.1, and postpone further results to future work.

\section{Preliminaries}

2.1. Polars and projection properties. For the standard notions that we recall in this subsection, see [3. Ch. 3, 6, and 11], together with [17, Ch. 4, §24]. Given any $\ell$-group $A$, the elements $x, y \in A$ are orthogonal, written $x \perp y$, if $|x| \wedge|y|=0$. For $T \subseteq A$, we set

$$
T^{\perp}:=\{x \in A \mid x \perp y \text { for all } y \in T\} ;
$$

we write $T^{\perp \perp}$ instead of $\left(T^{\perp}\right)^{\perp}$, and $x^{\perp}$ instead of $\{x\}^{\perp}$ for $x \in A$. A subset $S \subseteq A$ is a polar if it satisfies $S=S^{\perp \perp}$, or equivalently, if there exists $T \subseteq A$ such that $S=T^{\perp}$. When necessary, we will denote polars computed in $A$ by $T^{\perp_{A}}$. We write $\operatorname{Pol} A$ to denote the set of polars of $A$. Under the inclusion order, $\operatorname{Pol} A$ is a complete distributive lattice with $A=0^{\perp}$ as maximum, $\{0\}=A^{\perp}=0^{\perp \perp}$ as minimum, meets given by intersections, and joins given by $\bigvee S_{i}:=\left(\bigcup S_{i}\right)^{\perp \perp}$. It can be shown that $\mathrm{Pol} A$ is a complete Boolean algebra, with complementation given by the map $S \in \operatorname{Pol} A \mapsto S^{\perp} \in \operatorname{Pol} A$. In particular, for any subset $T \subseteq A$ we have $T^{\perp \perp \perp}=T^{\perp}$.

If $x \in A$, the set $x^{\perp \perp}$ is called the principal polar generated by $x$. Then $x^{\perp \perp} \in \operatorname{Pol} A$, and $x^{\perp \perp}=\bigcap_{x \in S \in \operatorname{Pol} A} S$, that is, $x^{\perp \perp}$ is the inclusion-smallest polar containing $\{x\}$. We write $\operatorname{Pol}_{\mathrm{p}} A$ to denote the set of principal polars of $A$; it 
is a sublattice of $\operatorname{Pol} A$, because of the identities

$$
\begin{aligned}
& (x \wedge y)^{\perp \perp}=x^{\perp \perp} \cap y^{\perp \perp} \\
& (x \vee y)^{\perp \perp}=x^{\perp \perp} \vee y^{\perp \perp},
\end{aligned}
$$

which hold for each $x, y \in A^{+}$. Further, the minimum $0^{\perp \perp}$ of $\operatorname{Pol} A$ lies in $\operatorname{Pol}_{p} A$. However, the maximum $A=0^{\perp}$ of $\mathrm{Pol} A$ need not be a principal polar: in fact, this happens precisely when $A$ has a weak unit $w$, and in that case $A=w^{\perp \perp}$. Even when $A$ has a weak unit, $\operatorname{Pol}_{\mathrm{p}} A$ may fail to be a Boolean subalgebra of $\operatorname{Pol} A$, because the complement of a principal polar need not be principal.

An ideal $I \subseteq A$ is closed, or is a band, if for each $S \subseteq I$ such that $\bigvee S$ exists in $A$, we have $\bigvee S \in I$. It can be shown that each polar is a band; for the converse, we have the important

Lemma 2.1. An $\ell$-group $A$ is such that its polars coincide with its bands if, and only if, $A$ is Archimedean.

Proof. [3, 11.1.10].

A band $I \subseteq A$ is a projection band if there is a product splitting $A \cong I \times I^{\perp}$.

Definition 2.2 (Cf. [17, 24.8]). An $\ell$-group $A$ is said to have the principal projection property, or to be projectable, if each principal band of $A$ is a projection band. Further, $A$ is said to have the projection property, or to be strongly projectable, if each band of $G$ is a projection band.

We recall here a standard fact:

Lemma 2.3. An $\ell$-group with the principal projection property must be Archimedean.

Proof. The (easy) proof for vector lattices given in [17, 24.9] works for $\ell$-groups without changes.

Remark 2.4. Projection properties are a classical topic in the theory of vector lattices, see [17, Ch. 4]. In the literature on $\ell$-groups, it is standard to call $A$ projectable when each of its principal polars is a cardinal summand (i.e. a factor of a product splitting) of $A$, and strongly projectable when the same holds for all polars. Thus, we see from Lemmas 2.3 and 2.1 that an $\ell$-group $A$ has the principal projection property if, and only if, it is projectable in the present sense; and that it has the projection property if, and only if, it is strongly projectable in the present sense. Cf. also [3, 7.5]. This explains the alternative terminologies in Definition 2.2. In the rest of this paper we shall use the terminology projectable.

A component of the unit $u$ is an element $\chi \in G$ such that $\chi \vee(u-\chi)=u$ and $\chi \wedge(u-\chi)=0$. It is well known that this entails the existence of a product splitting $G \cong \chi^{\perp} \times \chi^{\perp \perp}$. Conversely, if $G \cong A \times B$ in $\mathbf{U}$, then there is a unique $\chi \in G-$ namely, the image in $G$ of the unit of $B$ under the unital isomorphism $G \cong A \times B$ - that is a component of the unit $u$ such that $A \cong \chi^{\perp}$ and $B \cong \chi^{\perp \perp}$. We use these elementary facts without further justification throughout.

Finally, we recall the version of Freudenthal's Spectral Theorem that we will use.

Theorem 2.5. Let $R$ be a Riesz space that is projectable and has a unit u. For $v \in R$, set $\|v\|_{u}:=\inf \{\lambda \in \mathbb{R} \mid \lambda \geqslant 0$ and $\lambda u \geqslant|v|\}$. Then $\|v\|_{u}$ is a norm on $R$. For each $v \in R$ there is a sequence $\left\{c_{i}\right\}_{i \geqslant 1} \subseteq R$ of linear combinations of components of $u$ that converges to $v$ uniformly in the norm $\|\cdot\|_{u}$. 
Proof. See [17, 40.2].

2.2. Essential extensions and the projectable hull. A monomorphism $\iota:(G, u)$ $\hookrightarrow(H, v)$ in $\mathbf{U}$ will be referred to as an extension (of $G$ by $H$ ). The extension is essential if whenever a $\mathbf{U}$-morphism $f:(H, v) \rightarrow(A, a)$ is such that the composition $f \circ \iota$ is monic, then $f$ is monic. Amongst several well-known characterisations of essential extensions we shall use the following.

Lemma 2.6. Let $\iota:(G, u) \hookrightarrow(H, v)$ be a monomorphism in $\mathbf{U}$. The following are equivalent.

(1) The extension $\iota$ is essential.

(2) The map $\nu_{H}: P \in \operatorname{Pol} H \longmapsto P \cap \iota(G) \in \operatorname{Pol} \iota(G)$ is an isomorphism from the Boolean algebra of polars of $H$ onto that of $\iota(G)$. The inverse isomorphism is the map $\nu_{H}^{-1}: Q \in \operatorname{Pol} \iota(G) \longmapsto Q^{\perp \perp_{H}} \in \operatorname{Pol} H$.

(3) For each $y \in H$ with $y>0$ there is $x \in G$ with $0<\iota(x)<n y$ for some integer $n>0$.

Proof. See [6, Prop. 3.1 and Thm. 3.7] and [7, §2].

Definition 2.7. An essential extension $\epsilon:(G, u) \hookrightarrow(K, w)$ in $\mathbf{U}$ is said to be a projectable hull if $K$ is projectable, and whenever $\iota:(G, u) \hookrightarrow(H, v)$ is another essential extension with $H$ projectable, there exists an injective $\ell$-homomorphism $\varphi:(K, w) \rightarrow(H, v)$ in $\mathbf{U}$ that makes the following diagram commute.

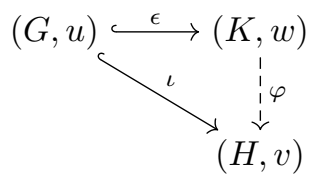

It turns out that the $\ell$-homomorphism $\varphi$ in the preceding definition is automatically an essential extension. Also note that a projectable hull is unique up to an isomorphism in $\mathbf{U}$.

Remark 2.8. Through the general treatment in [2, hulls related to projectability properties can and have been fruitfully investigated at the level of all lattice-ordered (not necessarily Abelian) groups, with no assumption on the existence of units. In particular, any lattice-ordered group turns out to have a strongly projectable hull in this generalised sense, 2, Thm. 2.25], which agrees with the usual one in the representable case.

2.3. The Yosida representation: the case of strong units. For $X$ a topological space, recall that a subset $S \subseteq \mathrm{C}(X)$ is said to separate the points of $X$ if for any $x \neq y \in X$ there is $f \in S$ with $f(x) \neq f(y)$. The next result summarises the classical Yosida representation; everything is rooted and essentially proved in 21.

Theorem 2.9 (The Yosida Representation). Recall that $(G, u)$ is a $\mathbf{U}$-object with maximal spectral space $X_{G}$.

(a) For each $\mathfrak{m} \in X_{G}$, there exists a unique monomorphism

$$
\iota_{\mathfrak{m}}:(G / \mathfrak{m}, u / \mathfrak{m}) \hookrightarrow(\mathbb{R}, 1)
$$

in $\mathbf{U}$. Upon setting

$$
\widehat{g}(\mathfrak{m}):=\iota_{\mathfrak{m}}(g / \mathfrak{m}) \in \mathbb{R},
$$


each $g \in G$ induces a function

$$
\widehat{g}: X_{G} \rightarrow \mathbb{R}
$$

that is continuous with respect to the spectral topology on the domain and the Euclidean topology on the co-domain.

(b) The map

$$
\widehat{\cdot}:(G, u) \longrightarrow\left(\mathrm{C}\left(X_{G}\right), 1_{X_{G}}\right)
$$

given by (a) is a monomorphism in $\mathbf{U}$ whose image $\widehat{G} \subseteq \mathrm{C}\left(X_{G}\right)$ separates the points of $X_{G}$.

(c) $X_{G}$ is unique up to a unique homeomorphism with respect to its properties. More explicitly, if $Y$ is any compact Hausdorff space, and $e:(G, u) \hookrightarrow$ $\left(\mathrm{C}(Y), 1_{Y}\right)$ is any monomorphism in $\mathbf{U}$ whose image $e(G) \subseteq \mathrm{C}(Y)$ separates the points of $Y$, then there exists a unique homeomorphism $f: Y \rightarrow$ $X_{G}$ such that $(e(g))(y)=\widehat{g}(f(y))$ for all $g \in G$ and $y \in Y$.

Remark 2.10. For the more general Yosida representation in the category of $\ell$ groups equipped with a weak unit, see the standard reference [15].

Remark 2.11. Let us explicitly observe that components of the unit $1_{X_{G}}$ in $\mathrm{C}\left(X_{G}\right)$ are precisely the characteristic functions $X_{G} \rightarrow \mathbb{R}$, i.e. the continuous functions with range contained in $\{0,1\}$.

\section{RePRESENTING AN $\ell$-GROUP ON ITS MINIMAL SPECTRUM}

Recall that $Z_{G}$ denotes the minimal spectral space of the $\mathbf{U}$-object $(G, u)$. We show in this section that $G$ may be represented as an $\ell$-subgroup of $\mathrm{C}\left(w_{G} Z_{G}\right)$ for the zero-dimensional Wallman compactification $w_{G} Z_{G}$ of $Z_{G}$, which we describe below. Before dealing with the general case, let us pause to recall that compactness of $Z_{G}$ is equivalent to a complementation property of $G$.

Definition 3.1 (9]). An $\ell$-group $A$ is complemented if for each $x \in A$ there exists $y \in A$ such that $|x| \wedge|y|=0$ and $|x| \vee|y| \neq 0$ is a weak unit of $A$.

Throughout we write $\cdot \backslash \cdot$ for set-theoretic difference. We recall $\mathbb{S}_{m}=\operatorname{Min} A \backslash \mathbb{V}_{m}(g)$. Conrad and Martinez [8] proved the equivalence of (i) and (ii) below.

Lemma 3.2. For an $\ell$-group $A$, the following are equivalent.

(i) $A$ is complemented.

(ii) $\operatorname{Min} A$ is compact.

(iii) There exists a weak unit in $A$, the lattice $\mathrm{Pol}_{\mathrm{p}} A$ is bounded, and the inclusion map $\mathrm{Pol}_{\mathrm{p}} A \hookrightarrow \operatorname{Pol} A$ is a homomorphism of Boolean algebras.

(iv) The distributive lattice $\left(\left\{\mathbb{V}_{m}(g)\right\}_{g \in A}, \cap, \cup\right)$ is a Boolean algebra, i.e. for all $g \in A$ there is $f \in A$ such that $\mathbb{S}_{m}(g)=\mathbb{V}_{m}(f)$.

Proof. ( $\mathrm{i} \Leftrightarrow \mathrm{ii}$ ) is [8, 2.2]. ( $\mathrm{i} \Leftrightarrow$ iii) is also stated in passing in [8]; its proof is an elementary application of (4) 5 ).

To prove (iii $\Leftrightarrow$ iv) we recall that $\bigcap_{\mathfrak{p} \in \operatorname{Min} G} \mathfrak{p}=\{0\}$ and that a prime $\mathfrak{p} \in \operatorname{Spec} A$ is minimal if and only if for all $g \in \mathfrak{p}$ there is an element $h \notin \mathfrak{p}$ with $h \perp g$ (see [3. 3.4.13]). We first show $\mathbb{V}_{m}(g)=\mathbb{V}_{m}\left(g^{\perp \perp}\right)$ for every $g \in A$. Since $g \in g^{\perp \perp}$, $\mathbb{V}_{m}\left(g^{\perp \perp}\right) \subseteq \mathbb{V}_{m}(g)$. Let $\mathfrak{p} \in \mathbb{V}_{m}(g)$. Since $g \in \mathfrak{p}$ and $\mathfrak{p} \in \operatorname{Min} A$, there exists $h \notin \mathfrak{p}$ such that $g \perp h$, whence $h \in g^{\perp}$. Let $f \in g^{\perp \perp}$. Then $f \in \mathfrak{p}$, because $|f| \wedge|h|=0$ and $\mathfrak{p}$ is prime. This ensures $\mathbb{V}_{m}\left(g^{\perp \perp}\right) \subseteq \mathbb{V}_{m}(g)$. 
(iv $\Rightarrow$ iii) Let $g \in A$ and $f \in A$ such that $\mathbb{S}_{m}(g)=\mathbb{V}_{m}(f)$. Then each $\mathfrak{p} \in \operatorname{Min} A$ is contained either in $\mathbb{V}_{m}(g)$ or in $\mathbb{V}_{m}(f)$. We show that $g^{\perp \perp}=f^{\perp}$.

Let $a \in f^{\perp}$. By primality, $a \in \mathfrak{p}$ for each $\mathfrak{p} \in \mathbb{V}_{m}(g)=\mathbb{S}_{m}(f)$, and hence $\mathbb{V}_{m}(g) \subseteq$ $\mathbb{V}_{m}(a)$. For each $b \in g^{\perp}, b \in \mathfrak{p}$ whenever $g \notin \mathfrak{p}$, whence $b \in \mathfrak{p}$ for each $\mathfrak{p} \in \mathbb{V}_{m}(f)$. Therefore, $|a| \wedge|b| \in \mathfrak{p}$ for each $\mathfrak{p} \in \operatorname{Min} A$, and hence $|a| \wedge|b|=0$. This ensures $a \in g^{\perp \perp}$, whence $g^{\perp \perp} \supseteq f^{\perp}$.

For the other inclusion, let $a \in g^{\perp \perp}$. By way of contradiction, suppose $|a| \wedge|f|=$ $c>0$. Then there exists $\mathfrak{p}_{c} \in \operatorname{Min} A$ such that $c \notin \mathfrak{p}_{c}$. As a consequence, $a \notin \mathfrak{p}_{c}$, $f \notin \mathfrak{p}_{c}$, and $g \in \mathfrak{p}_{c}$. By minimality of $\mathfrak{p}$, there exists $b \notin \mathfrak{p}_{c}$ such that $b \perp g$. Since $|a| \wedge|b|=0 \in \mathfrak{p}_{c}$, then $a \in \mathfrak{p}_{c}$, a contradiction. Hence we proved $g^{\perp \perp} \subseteq f^{\perp}$.

As a consequence, $g^{\perp \perp \perp}=f^{\perp \perp}$, whence the principal polar $f^{\perp \perp}$ is the complement of $g^{\perp \perp}$ in $\operatorname{Pol}_{\mathrm{p}} A$. Moreover, by taking $g=0$, there exists $w \in A$ such that $A=0^{\perp \perp \perp}=w^{\perp \perp}$. Then $\operatorname{Pol}_{\mathrm{p}} A$ is bounded and a Boolean algebra, and, as a direct consequence of the definition, $w$ is a weak unit for $A$.

(iii $\Rightarrow$ iv) Let $g \in A$ and $f \in A$ such that $f^{\perp \perp}$ is the complement of $g^{\perp \perp}$ in $\operatorname{Pol}_{\mathrm{p}} A$. Then $g^{\perp \perp \perp}=f^{\perp \perp}$, and $g^{\perp \perp}=f^{\perp}$. We show $\mathbb{V}_{m}(f)=\mathbb{S}_{m}\left(f^{\perp}\right)$, whence $\mathbb{V}_{m}(f)=\mathbb{S}_{m}\left(g^{\perp \perp}\right)=\mathbb{S}_{m}(g)$. Let $\mathfrak{p} \in \mathbb{V}_{m}(f)$, then $f \in \mathfrak{p}$. Since $\mathfrak{p}$ is minimal, there is $h \in A$ such that $h \perp f$ and $h \notin \mathfrak{p}$. Hence $h \in f^{\perp}$ and $\mathfrak{p} \notin \mathbb{V}_{m}\left(f^{\perp}\right)$. This ensures $\mathbb{V}_{m}(f) \subseteq \mathbb{S}_{m}\left(f^{\perp}\right)$. For the other inclusion, let $\mathfrak{p} \in \mathbb{S}_{m}\left(f^{\perp}\right)$ and $h \notin \mathfrak{p}$ such that $h \perp f$. Since $|h| \wedge|f|=0$ the primality of $\mathfrak{p}$ ensures $f \in \mathfrak{p}$, whence $\mathfrak{p} \in \mathbb{V}_{m}(f)$. Therefore $\mathbb{S}_{m}\left(f^{\perp}\right) \subseteq \mathbb{V}_{m}(f)$ and the proof is complete.

Remark 3.3. Versions of Lemma 3.2 for commutative rings, distributive lattices, and vector lattices were proved in [16, 3.4], [19, Prop. 3.2], and [17, 37.4], respectively.

We now turn to the $w_{G}$-compactification. Recall [18, 4.4(a)] that a Wallman base of a Hausdorff space $X$ is a base $\mathscr{L}$ of closed sets for $X$ that is stable under finite intersections and unions (and thus contains, in particular, $\varnothing$ and $X$ ), is such that if $A \in \mathscr{L}$ and $x \in X \backslash A$ then there is $B \in \mathscr{L}$ with $x \in B$ and $A \cap B=\varnothing$, and is such that for $A, B \in \mathscr{L}$ satisfying $A \subseteq X \backslash B$ there exist $C, D \in \mathscr{L}$ with $A \subseteq X \backslash C \subseteq D \subseteq X \backslash B$. Given such a base $\mathscr{L}$, let $w_{\mathscr{L}} X$ denote the collection of inclusion-maximal lattice filters of $\mathscr{L}$. The collection of sets $\left\{\mathscr{F} \in w_{\mathscr{L}} X \mid A \in \mathscr{F}\right\}$, as $A$ ranges in $\mathscr{L}$, is a base for the closed sets of a topology on $w_{\mathscr{L}} X$. With this topology, $w_{\mathscr{L}} X$ is compact [18, 4.4(d)]. Given $x \in X$, set $\mathscr{U}_{x}:=\{A \in \mathscr{L} \mid x \in A\}$. Then $\mathscr{U}_{x} \in w_{\mathscr{L}} X$, and the map

$$
\begin{aligned}
X & \longrightarrow w_{\mathscr{L}} X \\
x \in X & \longmapsto \mathscr{U}_{x} \in w_{\mathscr{L}} X
\end{aligned}
$$

is a dense embedding, called the Wallman compactification of $X$ induced by $\mathscr{L}$.

For any zero-dimensional Hausdorff space $X$, we note that any collection of clopen sets $\mathscr{B}$ of $X$ which also forms a closed base for the space, is, almost trivially, a Wallman base for the space, and that the generated Wallman compactification $w_{\mathscr{B}} X$ is a zero-dimensional Hausdorff space [18, 4.7(b)].

Furthermore, we note that in the case where $X$ is zero-dimensional and $\mathscr{B}$ is a Wallman base for $X$ consisting of clopen sets, the (bounded) functions from the uniformly closed subring of $\mathrm{C}(X)$ generated by the characteristic functions for the Boolean algebra of clopen sets generated by $\mathscr{B}$ are exactly the functions which extend to $w_{\mathscr{B}} X[18,4 \mathrm{I} \& 4 \mathrm{~J}]$. 
We next identify $G$ with its Yosida representation $\widehat{G} \subseteq \mathrm{C}\left(X_{G}\right)$, as given by Theorem 2.9. Recall the map $\lambda: Z_{G} \rightarrow X_{G}$ as in (12). If $\widehat{g} \in \widehat{G}$, the assignment

$$
\widehat{g} \in \widehat{G} \stackrel{\mu}{\longmapsto} \widehat{g} \circ \lambda \in \mathrm{C}\left(Z_{G}\right)
$$

yields a unital homomorphism of $\ell$-groups $\mu: \widehat{G} \rightarrow \mathrm{C}\left(Z_{G}\right)$, and a straightforward computation confirms that $\mu$ is injective because $\lambda$ is surjective. We therefore obtain a representation of $G$ as

$$
\mu(\widehat{G}) \subseteq \mathrm{C}\left(Z_{G}\right) .
$$

Remark 3.4. We notice that the Yosida representation $\widehat{g}$ of $g$ is such that, for every $\mathfrak{m} \in X_{G}, \widehat{g}(\mathfrak{m})=0$ if, and only if, $g \in \mathfrak{m}$. This ensures the inclusion of zero sets

$$
\mathbb{V}_{m}(g) \subseteq\left\{\mathfrak{p} \in Z_{G} \mid \mu(\widehat{g})(\mathfrak{p})=\widehat{g}(\lambda(\mathfrak{p}))=0\right\}
$$

Hence

$$
\lambda^{-1}\left(\mathbb{S}_{M}(g)\right)=\mathbb{S}(\mu(\widehat{g})) \subseteq \mathbb{S}_{m}(g),
$$

where $\mathbb{S}(\mu(\widehat{g})):=Z_{G} \backslash \mu(\widehat{g})^{-1}(0)$ is the support of $\mu(\widehat{g})$ at $Z_{G}$, and $\lambda^{-1}\left(\mathbb{S}_{M}(g)\right)$ is the preimage of $\mathbb{S}_{M}(g)$ under $\lambda$.

We consider now the Boolean algebra $\mathscr{B}\left(Z_{G}\right)$ of subsets of $Z_{G}$ generated by the collection $\left\{\mathbb{V}_{m}(g)\right\}_{g \in G}$. By [3, 10.2.1], for every $g \in G, \mathbb{V}_{m}(g) \subseteq Z_{g}$ is a clopen set, hence all the elements of $\mathscr{B}\left(Z_{G}\right)$ are clopens of $Z_{G}$. Moreover, since $\left\{\mathbb{V}_{m}(g)\right\}_{g \in G} \subseteq \mathscr{B}\left(Z_{G}\right)$, they also form a closed base for $Z_{G}$, and hence $\mathscr{B}\left(Z_{G}\right)$ is a Wallman base of $Z_{G}$. We denote by

$$
w_{G} Z_{G}
$$

the Wallman compactification $w_{\mathscr{B}\left(Z_{G}\right)} Z_{G}$ given by $\mathscr{B}\left(Z_{G}\right)$. The Boolean algebra of all clopens of $w_{G} Z_{G}$, written $\operatorname{Cp}\left(w_{G} Z_{G}\right)$, can be identified with the elements of $\mathscr{B}\left(Z_{G}\right)$ :

$$
\mathscr{B}\left(Z_{G}\right) \cong \operatorname{Cp}\left(w_{G} Z_{G}\right) .
$$

Let $\mathrm{K}\left(Z_{G}\right) \subseteq \mathrm{C}\left(Z_{G}\right)$ be the Boolean algebra of characteristic functions for the elements of $\mathscr{B}\left(Z_{G}\right)$. Since each member of $\mathrm{K}\left(Z_{G}\right)$ extends uniquely to a member of the Boolean algebra $\mathrm{K}\left(w_{G} Z_{G}\right)$ of all characteristic functions in $\mathrm{C}\left(w_{G} Z_{G}\right)$ by [18, $4 \mathrm{G}$ ], we have the isomorphism of Boolean algebras

$$
\mathrm{K}\left(Z_{G}\right) \cong \mathrm{K}\left(w_{G} Z_{G}\right) .
$$

In summary, any two Boolean algebras in (10) and (11) are isomorphic, and their dual Stone space is (9).

Remark 3.5. It may happen that $w_{G} Z_{G}$ is strictly smaller than the Banaschewski compactification of $Z_{G}$, the largest zero-dimensional compactification usually denoted $\beta_{0} Z_{G}$. See Section 6 .

Lemma 3.6. With reference to the embedding (8), the uniform completion of the linear subspace of $\mathrm{C}\left(Z_{G}\right)$ generated by $\mathrm{K}\left(Z_{G}\right)$ contains $\mu(\widehat{G})$.

Proof. Let $V$ be the Riesz space generated by $\mu(\widehat{G}) \cup \mathrm{K}\left(Z_{G}\right)$ in $\mathrm{C}\left(Z_{G}\right)$.

Claim 3.7. The function $1_{Z_{G}}$ is a strong unit for $V$. 
Proof. For each $g \in G$ the function $\widehat{g}$ is bounded in $X_{G}$. Since the image of the function $\widehat{g} \circ \lambda$ coincides with the image of $\widehat{g}$, each element $\mu(\widehat{g}) \in \mu(\widehat{G})$ is bounded. The characteristic functions in $\mathrm{K}\left(Z_{G}\right)$ are bounded functions by definition. Therefore each element of $V$ is bounded, because the Riesz space operations preserve the property of being bounded. Hence $1_{Z_{G}}$ is a strong unit for $V$.

For each $g \in G$ let $\chi_{g} \in \mathrm{K}\left(Z_{G}\right)$ be the characteristic function of $\mathbb{S}_{m}(g)$.

Claim 3.8. $\chi_{g}^{\perp}=\mu(\widehat{g})^{\perp}$ in $V$.

Proof of Claim 3.8. Let $v \in V$ such that $v \in \chi_{g}^{\perp}$. Hence $v(\mathfrak{p}) \wedge \chi_{g}(\mathfrak{p})=0$ for every $\mathfrak{p} \in Z_{G}$. As a consequence, if $\chi_{g}(\mathfrak{p}) \neq 0$, then $v(\mathfrak{p})=0$. Therefore, whenever $\mu(\widehat{g})(\mathfrak{p}) \neq 0$, we have $v(\mathfrak{p})=0$ by Remark 3.4. Then $v(\mathfrak{p}) \wedge \mu(\widehat{g})(\mathfrak{p})=0$ for all $\mathfrak{p} \in Z_{G}$, whence $v \in \mu(\widehat{g})^{\perp}$. This proves the inclusion $\chi_{g}^{\perp} \subseteq \mu(\widehat{g})^{\perp}$.

For the other inclusion, suppose there exists $v \in V$ such that $v \in \mu(\widehat{g})^{\perp} \backslash \chi_{g}^{\perp}$. Then the supports $\mathbb{S}(v)$ and $\mathbb{S}(\mu(\widehat{g}))$ are disjoint, and there is $\mathfrak{p} \in Z_{G}$ such that $|v(\mathfrak{p})| \wedge\left|\chi_{g}(\mathfrak{p})\right| \neq 0$. By definition of $\chi_{g}, \chi_{g}^{-1}(1)=\mathbb{S}\left(\chi_{g}\right)=\mathbb{S}_{m}(g)$ and therefore $\mathfrak{p}$ is in $\mathbb{S}(v) \cap \mathbb{S}_{m}(g)$, which is open since $v$ is a continuous function on $Z_{G}$ and $\mathbb{S}_{m}(g)$ is a basic open subset of $Z_{G}$. Hence there exists a (nonempty) basic open set $\mathbb{S}_{m}(h)$ (with $0 \neq h \in G$ ) such that $\mathfrak{p} \in \mathbb{S}_{m}(h) \subseteq \mathbb{S}(v) \cap \mathbb{S}_{m}(g)$. Let $\mathfrak{m} \in \mathbb{S}_{M}(h)$. Then $h \notin \mathfrak{m}$, whence $h \notin \mathfrak{q}$ for every $\mathfrak{q} \in \lambda^{-1}(\mathfrak{m})$ and $\lambda^{-1}(\mathfrak{m}) \subseteq \mathbb{S}_{m}(h)$. Since $\mathbb{S}_{m}(h)$ is disjoint from $\mathbb{S}(\mu(\widehat{g}))$, and $\mathbb{S}(\mu(\widehat{g}))=\lambda^{-1}\left(\mathbb{S}_{M}(g)\right)$ by Remark 3.4 $\mathfrak{q} \notin \lambda^{-1}\left(\mathbb{S}_{M}(g)\right)$ for every $\mathfrak{q} \in \lambda^{-1}(\mathfrak{m})$. Then $\mathfrak{m} \in \mathbb{V}_{M}(g)$ and $g \in \mathfrak{m}$. This ensures $\mathbb{S}_{M}(h) \subseteq \mathbb{V}_{M}(g)$, and hence $|h| \wedge|g|=0$. By primality of $\mathfrak{p}$, either $h \in \mathfrak{p}$ or $g \in \mathfrak{p}$, in contradiction with $\mathfrak{p} \in \mathbb{S}_{m}(h) \subseteq \mathbb{S}_{m}(g)$. This ensures $v \in \chi_{g}^{\perp}$, whence $\mu(\widehat{g})^{\perp} \subseteq \chi_{g}^{\perp}$.

Claim 3.9. $V$ is projectable.

Proof of Claim 3.9. We need to show that each $v \in V$ induces a product splitting $V \cong v^{\perp \perp} \times v^{\perp}$. By Claim 3.8, $\chi_{g}^{\perp \perp}=\mu(\widehat{g})^{\perp \perp}$ for all $g \in G$. But since $\chi_{g}$ is a component of $1_{Z_{G}}$ (because $\chi_{g} \vee\left(1_{Z_{G}}-\chi_{g}\right)=1_{Z_{G}}$ and $\left.\chi_{g} \wedge\left(1_{Z_{G}}-\chi_{g}\right)=0\right)$, there is an induced splitting $V \cong \chi_{g}^{\perp \perp} \times \chi_{g}^{\perp}$. This shows that each element of $V$ that lies in the generating set $\mu(\widehat{G}) \cup \mathrm{K}\left(Z_{G}\right)$ induces the splitting above. A routine induction argument now shows that the splitting property is preserved by sums, meets and joins, and products by real scalars, thus settling the claim.

By the preceding claim, we may apply Freudenthal's Spectral Theorem 2.5 to $\left(V, 1_{Z_{G}}\right)$, and infer that each element of $\mu(\widehat{G})$ is a $1_{Z_{G}}$-uniform limit of a sequence of elements in the linear subspace of $\mathrm{C}\left(Z_{G}\right)$ generated by $\mathrm{K}\left(Z_{G}\right)$. Since the norm induced by $1_{Z_{G}}$ on $\mathrm{C}\left(Z_{G}\right)$ coincides with the supremum norm, this completes the proof.

Lemma 3.10. For each $g \in G$, there exists a unique continuous extension of $\mu(\widehat{g}) \in \mathrm{C}\left(Z_{G}\right)$ to an element $g^{\sharp} \in \mathrm{C}\left(w_{G} Z_{G}\right)$. That is, $g^{\sharp}$ is the unique such element whose restriction to $Z_{G}$ is $\mu(\widehat{g})$. In symbols,

$$
g^{\sharp}\left\lceil Z_{G}=\mu(\widehat{g}) .\right.
$$

Proof. Indeed, by Lemma 3.6 there is a sequence $\left\{c_{i}\right\}_{i \geqslant 1}$ of linear combinations of elements of $\mathrm{K}\left(Z_{G}\right)$ that converges uniformly to $\mu(\widehat{g})$. Each member of $\mathrm{K}\left(Z_{G}\right)$ extends uniquely to a member of $\mathrm{K}\left(w_{G} Z_{G}\right)[18,4 \mathrm{G}]$, and therefore each $c_{i}$ extends to a linear combination $k_{i}$ of elements of $\mathrm{K}\left(w_{G} Z_{G}\right)$. It is now elementary to check 
that $\left\{k_{i}\right\}_{i \geqslant 1}$ is a Cauchy sequence in $\mathrm{C}\left(w_{G} Z_{G}\right)$ because $\left\{c_{i}\right\}_{i \geqslant 1}$ is one in $\mathrm{C}\left(Z_{G}\right)$. Take $g^{\sharp}$ to be the limit of $\left\{k_{i}\right\}_{i \geqslant 1}$, which is of course a continuous function by the Uniform Limit Theorem. Finally, note that $g^{\sharp}$ has property (12) by construction, and is the unique member of $\mathrm{C}\left(w_{G} Z_{G}\right)$ with this property because $Z_{G}$ is dense in its $w_{G}$-compactification, and the codomain of the functions - namely, $\mathbb{R}$ - is Hausdorff.

In light of Lemma 3.10, the function

$$
. \sharp: G \hookrightarrow \mathrm{C}\left(w_{G} Z_{G}\right)
$$

that acts by $g \mapsto g^{\sharp}$ is injective. It is elementary that this embedding preserves the lattice and group structure of $G$, and is also unit-preserving. We have therefore proved:

Theorem 3.11. Each $\mathbf{U}$-object $(G, u)$ has a representation into $\mathrm{C}\left(w_{G} Z_{G}\right)$ as in (13).

Definition 3.12. We write $\mathscr{P}(G)$ for the $\ell$-subgroup of $\mathrm{C}\left(w_{G} Z_{G}\right)$ generated by

$$
G^{\sharp} \cup \mathrm{K}\left(w_{G} Z_{G}\right) \text {. }
$$

We further write

$$
\pi: G \hookrightarrow \mathscr{P}(G)
$$

for the $\mathbf{U}$-monomorphism of $G$ into $\mathscr{P}(G)$ obtained by restricting the codomain of (13) to $\mathscr{P}(G)$.

\section{Characterisation of the elements of $\mathscr{P}(G)$}

In this section we characterise the functions in $\mathrm{C}\left(w_{G} Z_{G}\right)$ that lie in $\mathscr{P}(G)$. We begin by preparing two lemmas.

Lemma 4.1. There is a homeomorphism $\operatorname{Max} \mathscr{P}(G) \cong w_{G} Z_{G}$.

Proof. Indeed, the characteristic functions $\mathrm{K}\left(w_{G} Z_{G}\right) \subseteq \mathscr{P}(G)$ separate the points of $w_{G} Z_{G}$, because the latter is zero-dimensional; now apply Yosida's Theorem 2.9.

Remark 4.2. The next lemma is a consequence of more general results, cf. [14, Proposition 2.4]. We provide a proof here, for the reader's convenience.

Lemma 4.3. Let $g \in G$, and let $\chi \in G$ be a component of the unit $u$. Let us identify $G$ with its Yosida representation $\widehat{G} \subseteq \mathrm{C}\left(X_{G}\right)$. The pointwise product $g \chi$ defined by $(g \chi)(x)=g(x) \chi(x)$ for each $x \in X_{G}$ is a continuous function, and hence an element of $\mathrm{C}\left(X_{G}\right)$. Then $g \chi \in \widehat{G}$.

Proof. (Skipping all trivialities, in this proof we identify isomorphism with equality without further warning.) Since $\chi$ is a component of $u$ we have a product splitting $\widehat{G}=\chi^{\perp} \times \chi^{\perp \perp}(\perp$ computed in $\widehat{G})$, and a corresponding disjoint union decomposition $X_{G}=A \sqcup B, A:=\chi^{-1}(0), B:=\chi^{-1}(1), A$ and $B$ disjoint clopens in $X_{G}$. Then, clearly, $\mathrm{C}\left(X_{G}\right)=\mathrm{C}(A) \times \mathrm{C}(B), \chi^{\perp} \subseteq \mathrm{C}(A)$, and $\chi^{\perp \perp} \subseteq \mathrm{C}(B)$. Now since $g \in \chi^{\perp} \times \chi^{\perp \perp}, g$ may be uniquely expressed as a sum $g_{1}+g_{2}, g_{i} \in \widehat{G}, g_{1} \in \chi^{\perp}$, $g_{2} \in \chi^{\perp \perp}$. Then $g$ and $g_{2}$ agree over $B$, so that $g \chi=g_{2} \chi=g_{2} \in \widehat{G}$, and the lemma is proved. 
Remark 4.4. Let $0 \leqslant g \in G$, and let $\chi \in G$ be a component of the unit $u$. Identifying $G$ with its Yosida representation $\widehat{G} \subseteq \mathrm{C}\left(X_{G}\right)$, we notice that the function $g$ is bounded on the support of the characteristic function $\chi$. Therefore, there exists a (unique minimal) integer $n \geqslant 0$ such that $g \leqslant n \chi$ holds on the support of $\chi$, and hence $g \chi=g \wedge n \chi$ holds in $G$. This yields an explicit representation of the product $g \chi$ discussed in Lemma 4.3, using only the operations of $G$. Any element $g \in G$, indeed, can be written as the difference $g^{+}-g^{-}$between its positive part $g^{+}:=g \vee 0$ and its negative part $g^{-}:=(-g) \vee 0$, with $0 \leqslant g^{+}, g^{-} \in G$. As a consequence, there exist two (unique minimal) integers $n_{+}, n_{-} \geqslant 0$ such that $g \chi=\left(g^{+} \wedge n_{+} \chi\right)-\left(g^{-} \wedge n_{-} \chi\right)$.

In the following, we use the product $g \chi$ for brevity, but each such occurrence may be replaced by the equivalent expression $\left(g^{+} \wedge n_{+} \chi\right)-\left(g^{-} \wedge n_{-} \chi\right)$.

By a partition of unity in a $\mathbf{U}$-object $(G, u)$ we mean in this paper a finite family of non-zero elements $P:=\left\{\chi_{i}\right\}_{i=1}^{l}$ of $G$ such that $\sum_{i=1}^{l} \chi_{i}=u$, and $\chi_{i} \wedge \chi_{j}=0$ whenever $i \neq j$. It is elementary that each $\chi_{i}$ is a component of $u$. It follows that, in the Yosida representation $\widehat{G}$ of $G$, each $\widehat{\chi}_{i}$ is a characteristic function.

We can now prove:

Theorem 4.5. For each $e \in \mathrm{C}\left(w_{G} Z_{G}\right)$, the following are equivalent.

(1) $e \in \mathscr{P}(G)$.

(2) There exists a partition of unity $\chi_{1}, \ldots, \chi_{l}$ in $\mathscr{P}(G)$ - equivalently, in $\mathrm{C}\left(w_{G} Z_{G}\right)$ - along with elements $a_{1}, \ldots, a_{l} \in G$, such that

$$
e=\sum_{i=1}^{l} a_{i}^{\sharp} \chi_{i},
$$

where $\sharp$ is the embedding (13), and $a_{i}^{\sharp} \chi_{i}$ denotes the pointwise product of $a_{i}^{\sharp}$ and $\chi_{i}$ in $\mathrm{C}\left(w_{G} Z_{G}\right)$.

Proof. First, let us explicitly note that $\mathscr{P}(G)$ and $\mathrm{C}\left(w_{G} Z_{G}\right)$ have the same collection of partitions of unity because $\mathrm{K}\left(w_{G} Z_{G}\right) \subseteq \mathscr{P}(G)$.

(11) $\Rightarrow$ (2) Recall that $\mathscr{P}(G)$ is the $\ell$-subgroup of $\mathrm{C}\left(w_{G} Z_{G}\right)$ generated by the set (14). Hence by the elementary theory of lattice-groups we can write $e$ as

$$
\bigwedge_{i \in I} \bigvee_{j \in J}\left(g_{i j}^{\sharp}+c_{i j} k_{i j}\right)
$$

where $I$ and $J$ are finite sets of indices, at least one of which is non-empty, $g_{i j} \in G$, $c_{i j} \in \mathbb{Z}$ and $k_{i j} \in \mathrm{K}\left(w_{G} Z_{G}\right)$. Now for each $k_{i j}$, we obtain associated clopen subsets of $w_{G} Z_{G}$, namely their supports and their complements. This (necessarily nonempty) collection of clopens obviously covers $w_{G} Z_{G}$. It is elementary that we can refine this cover into a finite partition $\left\{D_{m}\right\}_{m \in M}$ of clopens of the space by taking intersections and set-theoretic differences.

On each $D_{m}$, each $k_{i j}$ is constant - either zero or one - by construction. Let us define the element $\delta_{i j}^{m} \in G$ by setting

$$
\delta_{i j}^{m}:= \begin{cases}u & \text { if } D_{m} \subseteq k_{i j}^{-1}(1), \\ 0 & \text { if } D_{m} \subseteq k_{i j}^{-1}(0) .\end{cases}
$$


Now consider the element of $\mathscr{P}(G)$

$$
e_{m}:=\bigwedge_{i \in I} \bigvee_{j \in J}\left(g_{i j}^{\sharp}+c_{i j}\left(\delta_{i j}^{m}\right)^{\sharp}\right) .
$$

Observe that the function $e_{m}$ agrees over $D_{m}$ with the function e, for each $m \in M$. This follows immediately from our definition of $\delta_{i j}^{m}$ in (17) above. Moreover, since $\sharp$ is an $\ell$-homomorphism, we have

$$
e_{m}=\left(\bigwedge_{i \in I} \bigvee_{j \in J}\left(g_{i j}+c_{i j} \delta_{i j}^{m}\right)\right)^{\sharp}=a_{m}^{\sharp},
$$

where $a_{m}:=\bigwedge_{i \in I} \bigvee_{j \in J}\left(g_{i j}+c_{i j} \delta_{i j}^{m}\right) \in G$. Hence, if we let $\chi_{m}$ be the characteristic function of $D_{m}$, we conclude

$$
e=\sum_{m \in M} a_{m}^{\sharp} \chi_{m}
$$

as was to be shown.

(22) $\Rightarrow$ (11) This follows at once from Lemmas 4.1 and 4.3 .

\section{Construction of the projectable hull}

Our final aim is to show that the embedding (13) provides a description of the projectable hull of $G$. This is our main result:

Theorem 5.1. For any $\mathbf{U}$-object $(G, u)$, the embedding $\pi: G \hookrightarrow \mathscr{P}(G)$ as in (15) is the projectable hull of $G$.

Proof. The proof that $\mathscr{P}(G)$ is projectable is identical to that of Claim 3.9

To prove that the map $\pi$ is an essential extension, we verify (3) in Lemma 2.6.

Pick $0<e \in \mathscr{P}(G)$, and express it as $e=\sum_{i=1}^{l} a_{i}^{\sharp} \chi_{i}$ by Theorem 4.5, for a partition of unity $\left\{\chi_{i}\right\}_{i=1}^{l}$ in $\mathscr{P}(G)$ and elements $\left\{a_{i}\right\}_{i=1}^{l}$ in $G$. Since $e>0$, we must have $a_{i}^{\sharp} \chi_{i} \geqslant 0$ for each $i$, and $a_{i_{0}}^{\sharp} \chi_{i_{0}}>0$ for some $i_{0}$. It is enough to show that there is $h \in G$ such that $0<h^{\sharp} \leqslant n a_{i_{0}}^{\sharp} \chi_{i_{0}}$, for then $0<h^{\sharp} \leqslant n e$ follows easily. By (11), we can identify the characteristic functions in $\mathrm{K}\left(w_{G} Z_{G}\right)$ with the elements of $\mathrm{K}\left(Z_{G}\right)$. Set $a:=a_{i_{0}}$ and $\chi:=\chi_{i_{0}} \wedge \chi_{a}$, where $\chi_{a} \in \mathrm{K}\left(Z_{G}\right)$ is the characteristic function of $\mathbb{S}_{m}(a)$. Since the support $\mathbb{S}(\chi)=\chi^{-1}(1)$ is clopen and nonempty (because $a^{\sharp} \chi \neq 0$ ), there exists $0<g \in G$ such that the basic open set $\mathbb{S}_{m}(g)$ is nonempty and contained in $\mathbb{S}(\chi) \subseteq \mathbb{S}_{m}(a)$. Hence $h:=g \wedge a>0$, and $\mathbb{S}\left(h^{\sharp}\right) \subseteq \mathbb{S}_{m}(h) \subseteq \mathbb{S}(\chi)$. We now claim that $h^{\sharp} \leqslant a^{\sharp} \chi$. It is enough to prove that the inequality holds for a point $x \in w_{G} Z_{G}$ in the support of $\chi$, where $\chi(x)=1$. Since $\sharp$ is an $\ell$-homomorphism, $h^{\sharp}=a^{\sharp} \wedge g^{\sharp}>0$. If $a^{\sharp}(x) \leqslant g^{\sharp}(x)$, then $h^{\sharp}(x)=a^{\sharp}(x)$, and the inequality holds. Otherwise, we have $h^{\sharp}(x)=g^{\sharp}(x)<a^{\sharp}(x)$. This settles the claim and completes the proof that $\pi$ is essential.

To show $\mathscr{P}(G)$ is a hull, it suffices to show that given the (unital) essential embedding $\iota$ into $H$ there exists an (automatically essential and unital) embedding 
$\varphi$ making the diagram below commute:

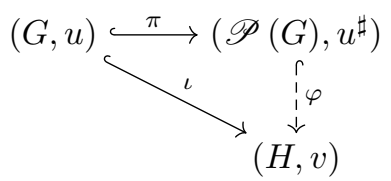

We define a function $\varphi: \mathscr{P}(G) \rightarrow H$ as follows. First we set

$$
\varphi\left(a^{\sharp}\right):=\iota(a) \text {, for each } a \in G \text {. }
$$

Since $H$ is projectable, for each $g \in G$ there is a unique component $\sigma_{g} \in H$ of the unit $v$ that satisfies $\iota(g)^{\perp \perp}=\sigma_{g}^{\perp \perp}$, where $\perp$ is computed in $H$. By (11), we identify the components of the unit $u^{\sharp}$ in $\mathscr{P}(G)$ with the characteristic functions in $\mathrm{K}\left(Z_{G}\right)$. Let $\chi_{g} \in \mathscr{P}(G)$ be the characteristic function of $\mathbb{S}_{m}(g)$ for $g \in G$. The analogous result of Claim 3.8 for $\mathscr{P}(G)$ ensures that $\chi_{g}^{\perp}=\pi(g)^{\perp}$ in $\mathscr{P}(G)$. We set

$$
\varphi\left(\chi_{g}\right):=\sigma_{g} .
$$

Let $\mathrm{K}(H)$ be the Boolean algebra of components of the unit $v$ in $H$.

Claim 5.2. The map $\varphi:\left\{\chi_{g}\right\}_{g \in G} \rightarrow \mathrm{K}(H)$ defined as in (19) is a lattice homomorphism.

Proof. Since $\iota$ is a unital homomorphism, trivially $\varphi\left(\chi_{0}\right)=0$ and $\varphi\left(\chi_{u}\right)=v$. Let $g_{1}, g_{2} \in G$. It is easy to check that $\chi_{g_{1}} \wedge \chi_{g_{2}}=\chi_{g_{1} \wedge g_{2}}$ and $\chi_{g_{1}} \vee \chi_{g_{2}}=\chi_{g_{1} \vee g_{2}}$. Let $\varphi\left(\chi_{g_{1}}\right)=\sigma_{g_{1}}, \varphi\left(\chi_{g_{2}}\right)=\sigma_{g_{2}}, \varphi\left(\chi_{g_{1} \wedge g_{2}}\right)=\sigma_{g_{1} \wedge g_{2}}$ and $\varphi\left(\chi_{g_{1} \vee g_{2}}\right)=\sigma_{g_{1} \vee g_{2}}$ as in (19). Hence, by (4) and (5),

$$
\begin{aligned}
\sigma_{g_{1} \wedge g_{2}}^{\perp \perp} & =\iota\left(g_{1} \wedge g_{2}\right)^{\perp \perp}=\left(\iota\left(g_{1}\right) \wedge \iota\left(g_{2}\right)\right)^{\perp \perp}= \\
& =\iota\left(g_{1}\right)^{\perp \perp} \cap \iota\left(g_{2}\right)^{\perp \perp}=\sigma_{g_{1}}^{\perp \perp} \cap \sigma_{g_{2}}^{\perp \perp}=\left(\sigma_{g_{1}} \wedge \sigma_{g_{2}}\right)^{\perp \perp}
\end{aligned}
$$

and

$$
\begin{aligned}
\sigma_{g_{1} \vee g_{2}}^{\perp \perp} & =\iota\left(g_{1} \vee g_{2}\right)^{\perp \perp}=\left(\iota\left(g_{1}\right) \vee \iota\left(g_{2}\right)\right)^{\perp \perp}= \\
& =\left(\iota\left(g_{1}\right)^{\perp \perp} \cup \iota\left(g_{2}\right)^{\perp \perp}\right)^{\perp \perp}=\left(\sigma_{g_{1}}^{\perp \perp} \cup \sigma_{g_{2}}^{\perp \perp}\right)^{\perp \perp}=\left(\sigma_{g_{1}} \vee \sigma_{g_{2}}\right)^{\perp \perp} .
\end{aligned}
$$

This ensures $\sigma_{g_{1} \wedge g_{2}}=\sigma_{g_{1}} \wedge \sigma_{g_{2}}$ and $\sigma_{g_{1} \vee g_{2}}=\sigma_{g_{1}} \vee \sigma_{g_{2}}$. Therefore $\varphi\left(\chi_{g_{1}} \wedge \chi_{g_{2}}\right)=$ $\varphi\left(\chi_{g_{1} \wedge g_{2}}\right)=\varphi\left(\chi_{g_{1}}\right) \wedge \varphi\left(\chi_{g_{2}}\right)$ and $\varphi\left(\chi_{g_{1}} \vee \chi_{g_{2}}\right)=\varphi\left(\chi_{g_{1} \vee g_{2}}\right)=\varphi\left(\chi_{g_{1}}\right) \vee \varphi\left(\chi_{g_{2}}\right)$, and the claim is proved.

The previous Claim 5.2, together with [1, Lemma 1 in Section V.4], ensures that $\varphi$ extends uniquely to a homomorphism $\varphi: \mathrm{K}\left(Z_{G}\right) \rightarrow \mathrm{K}(H)$ of Boolean algebras. Specifically, since the Boolean algebra $\mathrm{K}\left(Z_{G}\right)$ can be generated by the set $\left\{\chi_{g}\right\}_{g \in G}$ using the negation $u^{\sharp}$ - and the meet operation $\wedge$, the image of a general element of $\mathrm{K}\left(Z_{G}\right)$ under $\varphi$ can be inductively described as follows. Let $\chi_{1}, \chi_{2}, \alpha, \beta_{1}, \beta_{2} \in$ $\mathrm{K}\left(Z_{G}\right)$ such that $\chi_{1}=u^{\sharp}-\alpha$ and $\chi_{2}=\beta_{1} \wedge \beta_{2}$. Then

$$
\varphi\left(\chi_{1}\right)=v-\varphi(\alpha) \text { and } \varphi\left(\chi_{2}\right)=\varphi\left(\beta_{1}\right) \wedge \varphi\left(\beta_{2}\right) .
$$

Finally, for a general $e \in \mathscr{P}(G)$, we first write $e$ as in (16) using Theorem 4.5. and then, using (18, 200), we set

$$
\varphi(e):=\sum_{i=1}^{l} \varphi\left(a_{i}^{\sharp}\right) \varphi\left(\chi_{i}\right) .
$$


Since each product $\varphi\left(a_{i}^{\sharp}\right) \varphi\left(\chi_{i}\right)$ is an element of $H$ by Lemma 4.3. $\varphi(e)$ as in (21) is an element of $H$.

We next verify that $\varphi$ is a well-defined function. Given a decomposition (16) of $e \in \mathscr{P}(G)$ as in Theorem 4.5, suppose $e=\sum_{j=1}^{t} b_{j}^{\sharp} \xi_{j}$ is another such decomposition. It suffices to show that

$$
\sum_{i=1}^{l} \varphi\left(a_{i}^{\sharp}\right) \varphi\left(\chi_{i}\right)=\sum_{j=1}^{t} \varphi\left(b_{j}^{\sharp}\right) \varphi\left(\xi_{j}\right) .
$$

It is elementary to verify that the set $\left\{\chi_{i} \wedge \xi_{j} \mid \chi_{i} \wedge \xi_{j} \neq 0\right\}$ forms a partition of unity that refines both $\left\{\chi_{i}\right\}_{i=1}^{l}$ and $\left\{\xi_{j}\right\}_{j=1}^{t}$; that is, each $\chi_{i}$ and $\xi_{j}$ is a sum (or join, by pairwise disjointness) of elements $\chi_{i^{\prime}} \wedge \xi_{j^{\prime}} \neq 0$. It follows that $e$ can be expressed in two ways as

$$
e=\sum a_{i}^{\sharp}\left(\chi_{i} \wedge \xi_{j}\right)=\sum b_{j}^{\sharp}\left(\chi_{i} \wedge \xi_{j}\right),
$$

and hence $a_{i}^{\sharp}\left(\chi_{i} \wedge \xi_{j}\right)=b_{j}^{\sharp}\left(\chi_{i} \wedge \xi_{j}\right)$ for all $i, j$.

In the simplest case, we must prove:

Claim 5.3. Assuming $\chi \in \mathrm{K}\left(Z_{G}\right)$, suppose $a^{\sharp} \chi=b^{\sharp} \chi$. Then $\varphi\left(a^{\sharp} \chi\right)=\iota(a) \varphi(\chi)=$ $\iota(b) \varphi(\chi)=\varphi\left(b^{\sharp} \chi\right)$.

Proof. Let $\nu_{\mathscr{P}(G)}: \operatorname{Pol} \mathscr{P}(G) \rightarrow \operatorname{Pol} \pi(G)$ and $\nu_{H}^{-1}: \operatorname{Pol} \iota(G) \rightarrow \operatorname{Pol} H$ be the isomorphisms of Boolean algebras given in Lemma 2.6. Then also $\nu_{\pi}:=\pi^{-1} \circ$ $\nu_{\mathscr{P}(G)}: \operatorname{Pol} \mathscr{P}(G) \rightarrow \operatorname{Pol} G$ and $\nu_{\iota}:=\nu_{H}^{-1} \circ \iota: \operatorname{Pol} G \rightarrow \operatorname{Pol} H$ are isomorphisms of Boolean algebras. Since $\chi^{\perp \mathscr{P}(G)}$ is a polar, there exists a unique polar $P_{\chi} \in \operatorname{Pol} G$ such that $P_{\chi}^{\perp G}=\nu_{\pi}\left(\chi^{\perp \mathscr{P}(G)}\right)$. From $a^{\sharp} \chi=b^{\sharp} \chi$, we have $\pi(a-b)=\left(a^{\sharp}-b^{\sharp}\right) \in$

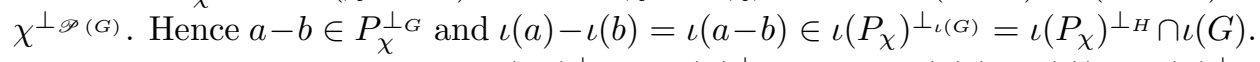
To complete the proof we show $\iota\left(P_{\chi}\right)^{\perp_{H}}=\varphi(\chi)^{\perp_{H}}$, whence $(\iota(a)-\iota(b)) \in \varphi(\chi)^{\perp_{H}}$ and $\iota(a) \varphi(\chi)=\iota(b) \varphi(\chi)$.

We proceed by induction on the structure of $\chi$ using (20). In the basic case we suppose $\chi:=\chi_{g}$, for $g \in G$. Then $\chi_{g}^{\perp \mathscr{P}(G)}=\pi(g)^{\perp \mathscr{P}(G)}$ by Claim 3.8, whence $P_{\chi}^{\perp_{G}}=\nu_{\pi}\left(\pi(g)^{\perp_{\mathscr{P}}(G)}\right)=\pi^{-1}\left(\nu_{\mathscr{P}(G)}\left(\pi(g)^{\perp_{\mathscr{P}}(G)}\right)\right)=\pi^{-1}\left(\pi(g)^{\perp_{\mathscr{P}}(G)} \cap \pi(G)\right)=$ $g^{\perp_{G}}=\left(g^{\perp^{\perp}}\right)^{\perp_{G}}$, and $P_{\chi}=g^{\perp \perp_{G}}$. By definition of $\varphi$ on $\left\{\chi_{g}\right\}_{g \in G}$, we have $\varphi(\chi)^{\perp_{H}}=\iota(g)^{\perp_{H}}=\iota\left(g^{\perp^{\perp}}\right)^{\perp_{H}}$, where the last equality is a direct consequence of the essentiality of $\iota$ (see [7, §2]).

We now consider the case $\chi:=u^{\sharp}-\alpha$, and suppose $\iota\left(P_{\alpha}\right)^{\perp_{H}}=\varphi(\alpha)^{\perp_{H}}$. Since $\chi$ and $\alpha$ are components of unity, $\chi^{\perp_{\mathscr{P}(G)}}=\alpha^{\perp \perp_{\mathscr{P}(G)}}$, whence $P_{\chi}^{\perp_{G}}=P_{\alpha}^{\perp \perp_{G}}$ and $\iota\left(P_{\chi}\right)^{\perp_{H}}=\iota\left(P_{\alpha}\right)^{\perp_{H}}$, because both $\nu_{\pi}$ and $\nu_{\iota}$ are isomorphisms of Boolean algebras. Since also $\varphi: \mathrm{K}\left(Z_{G}\right) \rightarrow \mathrm{K}(H)$ is an isomorphism of Boolean algebras, $\varphi(\chi)=v-\varphi(\alpha)$. Therefore, $\iota\left(P_{\chi}\right)^{\perp_{H}}=\iota\left(P_{\alpha}\right)^{\perp \perp_{H}}=\varphi(\alpha)^{\perp \perp_{H}}=\varphi(\chi)^{\perp_{H}}$.

The case $\chi:=\beta_{1} \wedge \beta_{2}$ is proved in a similar way. Suppose $\iota\left(P_{\beta_{1}}\right)^{\perp_{H}}=\varphi\left(\beta_{1}\right)^{\perp_{H}}$ and $\iota\left(P_{\beta_{2}}\right)^{\perp_{H}}=\varphi\left(\beta_{2}\right)^{\perp_{H}}$. By (44), $\chi^{\perp \perp_{\mathscr{P}(H)}}=\left(\beta_{1} \wedge \beta_{2}\right)^{\perp \perp \mathscr{P}(H)}=\beta_{1}^{\perp \perp_{\mathscr{P}}(H)} \cap \beta_{2}^{\perp \perp \mathscr{P}(H)}$, whence $\chi^{\perp \mathscr{P}(H)}=\left(\beta_{1}^{\perp \perp_{\mathscr{P}(H)}} \cap \beta_{2}^{\perp \perp_{\mathscr{P}(H)}}\right)^{\perp \mathscr{P}(H)}=\beta_{1}^{\perp \perp \mathscr{P}(H)} \vee \beta_{2}^{\perp \perp \mathscr{P}(H)}$. As a consequence, $P_{\chi}^{\perp \mathscr{P}(H)}=P_{\beta_{1}(H)}^{\perp \mathscr{P}(H} P_{\beta_{2}}^{\perp \mathscr{P}(H)}$ and $\iota\left(P_{\chi}\right)^{\perp_{H}}=\iota\left(P_{\beta_{1}}\right)^{\perp_{H}} \vee \iota\left(P_{\beta_{2}}\right)^{\perp_{H}}$. Since

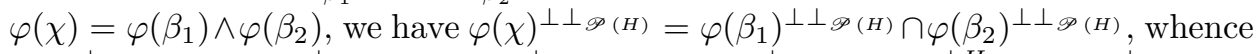
$\varphi(\chi)^{\perp \mathscr{P}(H)}=\varphi\left(\beta_{1}\right)^{\perp \mathscr{P}(H)} \vee \varphi\left(\beta_{2}\right)^{\perp \mathscr{P}(H)}=\iota\left(P_{\beta_{1}}\right)^{\perp_{H}} \vee \iota\left(P_{\beta_{2}}\right)^{\perp H}=\iota\left(P_{\chi}\right)^{\perp_{H}}$. The claim is settled. 
Since $\varphi: \mathrm{K}\left(Z_{G}\right) \rightarrow \mathrm{K}(H)$ is a homomorphism of Boolean algebras, $\varphi$ preserves partitions of unity and $\varphi(\chi \wedge \xi)=\varphi(\chi) \wedge \varphi(\xi)$. Therefore

$$
\begin{aligned}
& \sum \varphi\left(a_{i}^{\sharp}\right) \varphi\left(\chi_{i} \wedge \xi_{j}\right)=\sum \varphi\left(b_{j}^{\sharp}\right) \varphi\left(\chi_{i} \wedge \xi_{j}\right), \\
& \sum_{j} \varphi\left(a_{i}^{\sharp}\right) \varphi\left(\chi_{i} \wedge \xi_{j}\right)=\varphi\left(a_{i}^{\sharp}\right) \varphi\left(\chi_{i}\right) \text { for all } i \text { 's, } \\
& \sum_{i} \varphi\left(b_{j}^{\sharp}\right) \varphi\left(\chi_{i} \wedge \xi_{j}\right)=\varphi\left(b_{j}^{\sharp}\right) \varphi\left(\xi_{j}\right) \text { for all } j \text { 's. }
\end{aligned}
$$

These together prove (22).

The map $\varphi$ makes the diagram $($ * commute by construction. To show that it is an $\ell$-homomorphism one argues as follows. Given $e+f \in \mathscr{P}(G)$, to prove $\varphi(e+f)=\varphi(e)+\varphi(f)$, we first take decompositions of $e+f, e$ and $f$ as in (16) of Theorem 4.5. We then pick a joint refinement of the three partitions of unity involved. We finally proceed as in the preceding argument that shows $\varphi$ is well-defined. We omit the elementary details. The argument for the remaining operations is analogous.

To show $\varphi$ is injective, consider $e \neq f \in \mathscr{P}(G)$. Using again decompositions as in (16) of Theorem 4.5, and a common refinement of the associated partitions, we see that $e$ and $f$ must differ on some element of the common refinement. Injectivity of $\varphi$ then follows at once from the injectivity of $\iota$ and $\pi$.

\section{EXAMPLES}

We close the paper with two examples. Example 6.1 shows that the space $Z_{G}$ of minimal primes can fail to be compact even though its $G$-indexed compactification $w_{G} Z_{G}$ is actually $\beta_{0} Z_{G}$, the largest zero-dimensional compactification of $Z_{G}$. This happens when the base of clopens of $Z_{G}$ indexed by elements of $G$ is not a Boolean algebra - because it fails to be closed under complements - and yet it is large enough that the Boolean algebra it generates consists of all clopens of $Z_{G}$. We note in passing that the $\ell$-group in this first example can be shown to be finitely generated. Example 6.2 , by contrast, is an instance where the $G$-indexed compactification $w_{G} Z_{G}$ is strictly smaller than $\beta_{0} Z_{G}$. Here $G$ is of the form $\mathrm{C}(X)$ for $X$ a compact Hausdorff space.

Example 6.1. Let $\mathscr{M}$ be the set of continuous and piecewise linear functions $f:[0,1]^{2} \rightarrow \mathbb{R}$ with integer coefficients. This means that $f$ is continuous, and that there are finitely many triplets of integers $\left(a_{i}, b_{i}, c_{i}\right) \in \mathbb{Z}^{3}$ such that for all $(x, y) \in[0,1]^{2}$ we have $f(x, y)=a_{i} x+b_{i} y+c_{i}$ for some $i$. When equipped with the pointwise operations of minimum, maximum, and addition, $\mathscr{M}$ is an $\ell$-group. The function identically equal to 1 is a strong unit.

Let

$$
P:=\left\{(x, y) \in[0,1]^{2} \mid y=x^{2}\right\} \cup\left\{(x, y) \in[0,1]^{2} \mid y=0\right\},
$$

and let $\left(G, 1_{P}\right)$ be the unital $\ell$-group obtained by restricting each element of $\mathscr{M}$ to $P$, where $1_{P}: P \rightarrow \mathbb{R}$ is the function constantly equal to 1 . We now show that $Z_{G}:=\operatorname{Min} G$ fails to be compact. For this, consider the projection function $\pi_{y}:(x, y) \mapsto y$, along with the set of functions $h_{n}(0<n \in \mathbb{N})$ which are equal to $n x-1$ on the segment $\left\{(x, y) \in[0,1]^{2} \mid y=0\right.$ and $\left.x \in\left[\frac{1}{n}, 1\right]\right\}$ and to 0 elsewhere. These are elements of $G$. Then $\left\{\mathbb{S}_{m}\left(\pi_{y}\right)\right\} \cup\left\{\mathbb{S}_{m}\left(h_{n}\right)\right\}_{n>0}$ is an open cover of $Z_{G}$ without a finite subcover. According to Lemma 3.2 is not complemented. Indeed, 
by piecewise linearity and continuity, there can be no $f \in \mathscr{M}$ that restricts to $P$ so as to satisfy $\pi_{y}^{\perp \perp}=f^{\perp}$. By the same lemma, $\left\{\mathbb{V}_{m}(g)\right\}_{g \in G}$ is not a Boolean algebra. Indeed, it does not contain the complement $\mathbb{S}_{m}\left(\pi_{y}\right)$ of $\mathbb{V}_{m}\left(\pi_{y}\right)$. In particular, $\left\{\mathbb{V}_{m}(g)\right\}_{g \in G}$ is not the collection of all clopens of $Z_{G}$, because $\mathbb{S}_{m}\left(\pi_{y}\right)$ is clopen it is both a basic open set and the intersection of the closed sets in $\left\{\mathbb{V}_{m}\left(h_{n}\right)\right\}_{n>0}$. However, the Boolean algebra $\mathscr{B}\left(Z_{G}\right)$ generated by $\left\{\mathbb{V}_{m}(g)\right\}_{g \in G}$ is the collection of all clopens of $Z_{G}$, as can be seen by direct inspection. Therefore $\operatorname{Max} \mathscr{P}(G) \cong$ $w_{G} Z_{G}$ is homeomorphic to $\beta_{0} Z_{G}$, the maximal zero-dimensional compactification of $Z_{G}$.

Example 6.2. Let $D$ be the discrete space with $|D|=\aleph_{1}$, let $\alpha D=D \cup\{\alpha\}$ be the one-point compactification of $D$, and let $G=\mathrm{C}(\alpha D)$ so that $\operatorname{Max} G \cong \alpha D$. Then $Z_{G}:=\operatorname{Min} G$ can be identified with the collection of fixed ultrafilters on $D$ together with the free ultrafilters on $D$ which contain a countable subset. The basic closed sets of $Z_{G}$, of the form

$$
\left\{\mathscr{U} \in Z_{G} \mid g^{-1}(0) \in \mathscr{U}\right\},
$$

are actually clopen. We show that the Boolean algebra generated by these basic clopen sets is not the Boolean algebra of all clopens, but a proper subalgebra. For, let $D_{1}$ and $D_{2}$ be elements of a partition of $D$ with $\left|D_{i}\right|=\aleph_{1}, i=1,2$. Then we have an induced partition of $Z_{G}$ into two clopens:

$$
Z_{G}=\left\{\mathscr{U} \mid \exists C \in \mathscr{U} \text { with } C \subseteq D_{1}\right\} \biguplus\left\{\mathscr{U} \mid \exists C \in \mathscr{U} \text { with } C \subseteq D_{2}\right\} .
$$

Neither summand in the partition above is a basic closed set of $Z_{G}$. Therefore $\operatorname{Max} \mathscr{P}(G) \cong w_{G} Z_{G} ¥ \beta_{0} Z_{G}$.

Remark 6.3. In case $G=\mathrm{C}(X)$ for some compact Hausdorff space $X$, the main result of this paper is related to several others in the literature, and especially to the construction of zero-dimensional covers of $X$. Postponing fuller accounts to further research, we mention at least the following important connection. In [20, Vermeer constructs the basically disconnected cover of $X$ (in the more general case of a completely regular Hausdorff space) through an inverse limit [20, Theorem 4.4]. The inverse limit is defined by means of an auxiliary space $\Lambda_{1}(X)$ - itself a cover of $X-$ for which see in particular [20, Theorem 3.5]. Now, the results by Hager et al. in [14, Section 3] entail amongst other things that, in our notation, there is a homeomorphism $\operatorname{Max} \mathscr{P}(\mathrm{C}(X)) \cong \Lambda_{1}(X)$. By Lemma 4.1 we conclude $\Lambda_{1}(X) \cong w_{\mathrm{C}(X)} Z_{\mathrm{C}(X)}$. We thus obtain a description of Vermeer's cover $\Lambda_{1}(X)$ as the Wallman compactification of the minimal spectral space of $\mathrm{C}(X)$ described in (911) above. Contrast Vermeer's [20. Theorem 3.5], which describes $\Lambda_{1}(X)$ using regularised zero sets in the maximal spectral space $X$ of $\mathrm{C}(X)$. Further, the space $Z_{\mathrm{C}(X)}$ is compact if, and only if, $X$ is cozero-complemented, i.e. the lattice of cozero sets of $X$ is complemented; this is well-known and also follows from Lemma 3.2 In this case we have $w_{\mathrm{C}(X)} Z_{\mathrm{C}(X)} \cong Z_{\mathrm{C}(X)}$. Consequently, $\Lambda_{1}(X) \cong Z_{\mathrm{C}(X)}$ when $X$ is cozero-complemented, Vermeer's $\Lambda_{1}(X)$ is the minimal spectral space of $\mathrm{C}(X)$, and further work reveals Vermeer's cover $\Lambda_{1}(X) \rightarrow X$ in [20] as the "push-up" map $\lambda: Z_{\mathrm{C}(X)} \rightarrow X$ in (12).

\section{Acknowledgements.}

We gratefully acknowledge partial support by the Italian FIRB "Futuro in Ricerca" grant RBFR10DGUA. The grant partially supported a visit of R. N. Ball to the 
Università degli Studi di Milano, Italy, during which the fundamental ideas of the present paper were developed. We would also like to express our thanks to an anonymous referee for detecting a serious blunder in an earlier version of this paper, and for providing us with Example 6.2. The same referee pointed out to us the relevance of 20] and 14 for our results (cf. Remark 6.3). Finally, we are grateful to S. J. van Gool for pointing out to us that the use of the standard extension result 1. Lemma 1 in Section V.4] could shorten the proof of Theorem 5.1.

\section{REFERENCES}

[1] Raymond Balbes and Philip Dwinger. Distributive lattices. University of Missouri Press, Columbia, Mo., 1974.

[2] Richard N. Ball. The generalized orthocompletion and strongly projectable hull of a latticeordered group. Canad. J. Math., 34(3):621-661, 1982.

[3] Alain Bigard, Klaus Keimel, and Samuel Wolfenstein. Groupes et anneaux réticulés. Lecture Notes in Mathematics, Vol. 608. Springer-Verlag, Berlin, 1977.

[4] Roger D. Bleier. The SP-hull of a lattice-ordered group. Canad. J. Math., 26:866-878, 1974.

[5] Donald A. Chambless. Representation of the projectable and strongly projectable hulls of a lattice-ordered group. Proc. Amer. Math. Soc., 34:346-350, 1972.

[6] Paul Conrad. The essential closure of an Archimedean lattice-ordered group. Duke Math. J., 38:151-160, 1971.

[7] Paul Conrad. The hulls of representable l-groups and f-rings. J. Austral. Math. Soc., 16:385415, 1973. Collection of articles dedicated to the memory of Hanna Neumann, IV.

[8] Paul Conrad and Jorge Martinez. Complemented lattice-ordered groups. Indag. Math. (N.S.), $1(3): 281-297,1990$.

[9] Paul Conrad and Jorge Martinez. Very large subgroups of a lattice-ordered group. Comm. Algebra, 18(7):2063-2098, 1990.

[10] Michael R. Darnel. Theory of lattice-ordered groups, volume 187 of Monographs and Textbooks in Pure and Applied Mathematics. Marcel Dekker, Inc., New York, 1995.

[11] Hans Freudenthal. Teilweise geordnete Moduln. Proc. Akad. Wet. Amsterdam, 39:641-651, 1936.

[12] Leonard Gillman and Meyer Jerison. Rings of continuous functions. Springer-Verlag, New York, 1976. Reprint of the 1960 edition, Graduate Texts in Mathematics, No. 43.

[13] Andrew M. W. Glass. Partially ordered groups, volume 7 of Series in Algebra. World Scientific Publishing Co., Inc., River Edge, NJ, 1999.

[14] Anthony W. Hager, Chawne M. Kimber, and Warren Wm. McGovern. Weakly least integer closed groups. Rend. Circ. Mat. Palermo (2), 52(3):453-480, 2003.

[15] Anthony W. Hager and Lewis C. Robertson. Representing and ringifying a Riesz space. In Symposia Mathematica, Vol. XXI (Convegno sulle Misure su Gruppi e su Spazi Vettoriali, Convegno sui Gruppi e Anelli Ordinati, INDAM, Rome, 1975), pages 411-431. Academic Press, London, 1977.

[16] Melvin Henriksen and Meyer Jerison. The space of minimal prime ideals of a commutative ring. Trans. Amer. Math. Soc., 115:110-130, 1965.

[17] Wim A. J. Luxemburg and Adriaan C. Zaanen. Riesz spaces. Vol. I. North-Holland Publishing Co., Amsterdam, 1971. North-Holland Mathematical Library.

[18] Jack R. Porter and R. Grant Woods. Extensions and absolutes of Hausdorff spaces. SpringerVerlag, New York, 1988.

[19] Terence P. Speed. Some remarks on a class of distributive lattices. J. Austral. Math. Soc., 9:289-296, 1969.

[20] J. Vermeer. The smallest basically disconnected preimage of a space. Topology Appl., 17(3):217-232, 1984.

[21] Kôsaku Yosida. On vector lattice with a unit. Proc. Imp. Acad. Tokyo, 17:121-124, 1941.

[22] Kôsaku Yosida. On the representation of the vector lattice. Proc. Imp. Acad. Tokyo, 18:339342, 1942. 
(R. N. Ball) Department of Mathematics, University of Denver, Denver CO 80112, U.S.A.

(V. Marra, A. Pedrini) Dipartimento di Matematica Federigo Enriques, Università degli Studi di Milano, via Cesare Saldini 50, 20133 Milano, Italy.

(D. McNeill) Dipartimento di Scienze Teoriche e Applicate, Università degli Studi dell'Insubria, Via Mazzini 5, 21100 Varese, Italy.

E-mail address, R. N. Ball: rball@du.edu

E-mail address, V. Marra, A. Pedrini: \{vincenzo.marra, andrea.pedrini\}@unimi.it

E-mail address, D. McNeill: danmcne@gmail.com 